\title{
Miniaturized PIC multi-color laser engines for the life sciences
}

\begin{abstract}
Alireza Mashayekh, Thomas Klos, Sina Koch, Florian Merget, Douwe Geuzebroek, et al.
\end{abstract}

Alireza T. Mashayekh, Thomas Klos, Sina Koch, Florian Merget, Douwe Geuzebroek, Edwin Klein, Theo Veenstra, Martin Büscher, Patrick Leisching, Jeremy Witzens, "Miniaturized PIC multi-color laser engines for the life sciences," Proc. SPIE 10922, Smart Photonic and Optoelectronic Integrated Circuits XXI, 109221U (4 March 2019); doi: 10.1117/12.2507225 


\title{
Miniaturized PIC multi-color laser engines for the life sciences
}

\author{
Alireza T. Mashayekh*1, Thomas Klos², Sina Koch ${ }^{2}$, Florian Merget ${ }^{1}$, \\ Douwe Geuzebroek ${ }^{3}$, Edwin Klein ${ }^{3}$, Theo Veenstra ${ }^{3}$, Martin Büscher ${ }^{4}$, \\ Patrick Leisching ${ }^{2}$, Jeremy Witzens ${ }^{1}$ \\ ${ }^{1}$ RWTH Aachen University, Institute of Integrated Photonics, Aachen, Germany; \\ ${ }^{2}$ TOPTICA Photonics AG, Gräfelfing, Germany; \\ ${ }^{3}$ LioniX International BV, Enschede, The Netherlands; \\ ${ }^{4}$ Miltenyi Biotec, Bergisch Gladbach, Germany
}

\begin{abstract}
We are reporting on a Multi-Color Laser Engine (MLE) multiplexing four wavelengths (405 nm, $488 \mathrm{~nm}, 561 \mathrm{~nm}, 640$ $\mathrm{nm}$ ) by means of a Photonic Integrated Circuit (PIC) with Silicon Nitride ( $\mathrm{SiN}$ ) waveguides. Multiple building blocks are tested that allow manipulating the light in the waveguides to achieve fiber switching and variable optical attenuation. To slow down facet degradation and extend chip lifetime at near Ultra-Violet (UV) wavelengths (405 nm), a lateral endcap is implemented on chip and tested for reliability. Reasonable coupling and on-chip losses have been achieved in view of a practical use of the technology.
\end{abstract}

Keywords: Multi-color laser engine, silicon nitride technology, integrated photonic devices, photonic integrated circuits, variable optical attenuators, biophotonics, photonics in the life sciences.

\section{INTRODUCTION}

The SiN waveguide platform ${ }^{1,2}$ has favorable properties for visible wavelength PICs, in particular in view of servicing the needs of visible wavelength sensing and imaging in the life sciences. Sensing applications include for example Raman sensing $^{3}$, fluorescent sensing ${ }^{4}$, or refractive index sensing ${ }^{5}$. In addition to its wide transparency window, reaching from the UV to the Mid-Infrared (MIR) ${ }^{6}$, it has sufficiently low background luminescence to enable waveguide-based sensors to exhibit high sensitivity enhancements ${ }^{7}$. The moderate refractive index contrast between the $\mathrm{SiN}$ core and the silica cladding $(\Delta \mathrm{n} \sim 0.5)$ strikes a good compromise between compact routing and reduced scattering induced losses, with Near-Infrared (NIR) losses as low as $0.05 \mathrm{~dB} / \mathrm{m}$ in low confinement platforms ${ }^{8}$ (thin SiN films) and $0.05 \mathrm{~dB} / \mathrm{cm}$ in high confinement, densely routable platforms $s^{9}$ (thicker SiN films). The high damage threshold of SiN, vanishing Two-Photon Absorption (TPA) at NIR wavelengths and moderate third order nonlinearities also enable parametric light generation in high confinement waveguides ${ }^{10}$, with comb generation in the visible also recently demonstrated ${ }^{11}$. Lastly, compatibility of the SiN waveguide platform with standard Complementary-Metal-Oxide Semiconductor (CMOS) technology $y^{4,12}$ enables substantial cost reduction when sufficiently large fabrication scales are reached, so that a price point compatible with single-use lab-on-a-chip consumables may be reached ${ }^{13}$.

The focus of the work reported here is however rather on high-value instrumentation, namely Multi-Color Laser Engines (MLEs), a core element of cutting-edge biophotonic systems applied to confocal microscopy, fluorescent microscopy including super-resolution Stimulated Emission Depletion (STED) microscopy ${ }^{14}$ - flow cytometry ${ }^{15}$, optogenetics ${ }^{16}$, genetic analysis and DNA sequencing, to name just a few. These have in common the selective optical excitation of molecules - fluorophores, or, in the case of optogenetics, light-gated ion channels - with laser radiation falling within their absorption spectrum. Unambiguous identification of molecules or cellular subsets often requires jointly analyzing fluorescent signals from several fluorescent markers, so that MLEs are required to provide excitation wavelengths for several commercially available biocompatible fluorophores. A number of functionalities are required from MLEs in addition to sourcing the required wavelength $\mathrm{s}^{17}$ : Variable attenuation and/or digital intensity modulation in the $\mathrm{Hz}$ to $\mathrm{kHz}$ range are required for a number of applications such as optical trapping, lifetime imaging, or Fluorescence Recovery After Photobleaching (FRAP). Moreover, switching of the laser between two fiber outputs can be utilized e.g. to switch between scanning confocal microscopy and widefield illumination modes, e.g., for conventional fluorescence imaging.

* amashayekh@iph.rwth-aachen.de; phone +49 241 8020048; fax +49 241 8022212; iph.rwth-aachen.de

Smart Photonic and Optoelectronic Integrated Circuits XXI, edited by Sailing He, El-Hang Lee,

Proc. of SPIE Vol. 10922, 109221 U (c) The Authors. Published under a Creative

Commons Attribution CC-BY 3.0 License · doi: 10.1117/12.2507225 
MLEs assembled from discrete components require expensive components such as Acousto-Optic Tunable Filters (AOTFs), dichroic mirrors and fiber switches. Moreover, maintaining the long-term alignment of discretely assembled elements can be an engineering challenge and thus also a cost driver. Miniaturization of MLEs by taking advantage of SiN PICs also removes constraints in the architecture of the entire system: Rather than fiber coupling the MLE to the instrument, it would then be practicable to directly flange the miniaturized MLE onto the instrument, removing e.g. the need for interposed fibers. This then opens the potential for further innovation, such as directly beam forming the light on the PIC prior to free space coupling to the instrument ${ }^{18}$. Further miniaturization can be obtained by replacing Diode Pumped Solid State (DPSS) lasers by semiconductor diode lasers wherever possible. While this also enables direct modulation of the laser, the need for external modulation/variable attenuation is not fully removed, as turning down the diode lasers to very low power levels can also result in substantially increased noise ${ }^{19}$. The capability of attenuating the laser radiation inside the PIC, even with a finite extinction, thus increases the minimum power levels to which lasers have to be driven and will thus result in an improved noise performance.

To implement the PICs for the envisioned MLEs, we opted for the TriPleX ${ }^{\mathrm{TM}}$ platform developed by LioniX Int., whose capabilities have been recently extended to the visible range ${ }^{18}$ in the framework of the project PIX4life, funded by the European Union to support the growing application space of $\mathrm{SiN}$ based visible wavelength PICs in the life sciences. This platform is expected to be accessible through open access Multi-Project Wafer (MPW) fabrication runs in the future.

The currently available visible wavelength TriPleX platform consists in relatively low confinement waveguides formed by fully etching a single stoichiometric $\mathrm{Si}_{3} \mathrm{~N}_{4}$ film deposited by Low Pressure Vapor Deposition (LPCVD) onto oxidized Silicon wafers and later top-clad with deposited $\mathrm{SiO}_{2}$ (the single stripe geometry ${ }^{18}$ ). The material system has a wide transparency range starting at $400 \mathrm{~nm}$ and reaching up to $2.35 \mu \mathrm{m}$, as limited by absorption through $\mathrm{SiO}_{2}$ for the larger wavelengths. The lower wavelength cutoff is very sensitive to material quality, as non-stoichiometric silicon rich materials in particular result in higher absorption at the shorter wavelengths ${ }^{20}$. Thus, this platform allows covering the entire visible range used to excite fluorophores commonly utilized in the life sciences. As a particularity of the technology, SiN films are relatively thin (few tens of $\mathrm{nm}^{18}$ ), resulting in low confinement constraining e.g. the lower bending radii, but also facilitating matching of edge couplers to the beams emitted or received by standard visible wavelength optical fibers. While, without further material integration, the $\mathrm{SiN} / \mathrm{SiO}_{2}$ material system only allows relatively slow refractive index tuning via the thermo-optic effect ${ }^{22}$, integration of ferroelectric materials such as lead zirconate titanate (PZT) and application of stress via piezoelectric properties ${ }^{23}$ or direct refractive index change via the Pockels effect ${ }^{24}$ has yielded faster modulation schemes. Here we utilize thermal phase shifters adequate for the targeted $\mathrm{kHz}$ modulation speeds.

In previous works, we have reported adequate edge coupling to the developed PIC technology, both from fibers as well as from free space collimated beams inside an assembled optical module ${ }^{25}$. We have also introduced a low-loss multiplexer device concept adjustable to target wavelengths in the entire visible range and capable of combining the targeted $405 \mathrm{~nm}$, $488 \mathrm{~nm}, 561 \mathrm{~nm}$, and $640 \mathrm{~nm}$ wavelengths ${ }^{25}$. Here, we report on the complete characterization of one of the developed MLE system chips combining multiplexing with wavelength selective variable optical attenuation. Module assembly of this chip, as well as characteristics of other MLE chips additionally incorporating switching between output fibers or beam forming, will be reported at a later time.

Section 2 describes the PIC based MLE concept, including chip layout and the module assembly scheme, as well as the overall characteristics of the photonic system chip. Section 3 covers simulation and characterization results for individual on-chip building blocks - chip interfaces, multiplexer stages and Mach-Zehnder Interferometer (MZI) based Variable Optical Attenuators (VOAs) as individually measured from break-out structures on a test chip.

\section{PIC BASED MULTI-COLOUR LASER ENGINE}

A typical commercial MLE from TOPTICA is depicted in Figure 1(a). Four wavelengths, $405 \mathrm{~nm}, 488 \mathrm{~nm}, 561 \mathrm{~nm}$, and $640 \mathrm{~nm}$, are combined with dichroic mirrors, modulated by an AOTF, and sent through a fiber switch allowing rapid switching between different illumination modes. Constant Optical Output Levels (COOL) with Auto-Calibration (AC) are obtained with TOPTICA's proprietary $\mathrm{COOL}^{\mathrm{AC}}$ units $^{26}$ that allow adaptively steering the beam. Figure $1(\mathrm{~b})$ is a representation of the targeted MLE implementation with a SiN PIC. Light is coupled through focusing lenses to input edge couplers. On-chip, thermally tuned VOAs adjust the light intensity independently for each wavelength, after which the four are multiplexed by means of cascaded Wavelength Combiners (WLC). In an extended version of the chip, the VOAs 
have two complementary output ports that are sent to independent sets of wavelength combiners and coupled to one out of two fibers at the output, fulfilling all the functionalities of the commercial MLE represented in Fig. 1(a). While the lasers are free space coupled to the input of the chip with a set of lenses (see Section 2.1), light is directly edge coupled to fibers at the output by attaching a fiber array to the optical subassembly ${ }^{27}$.
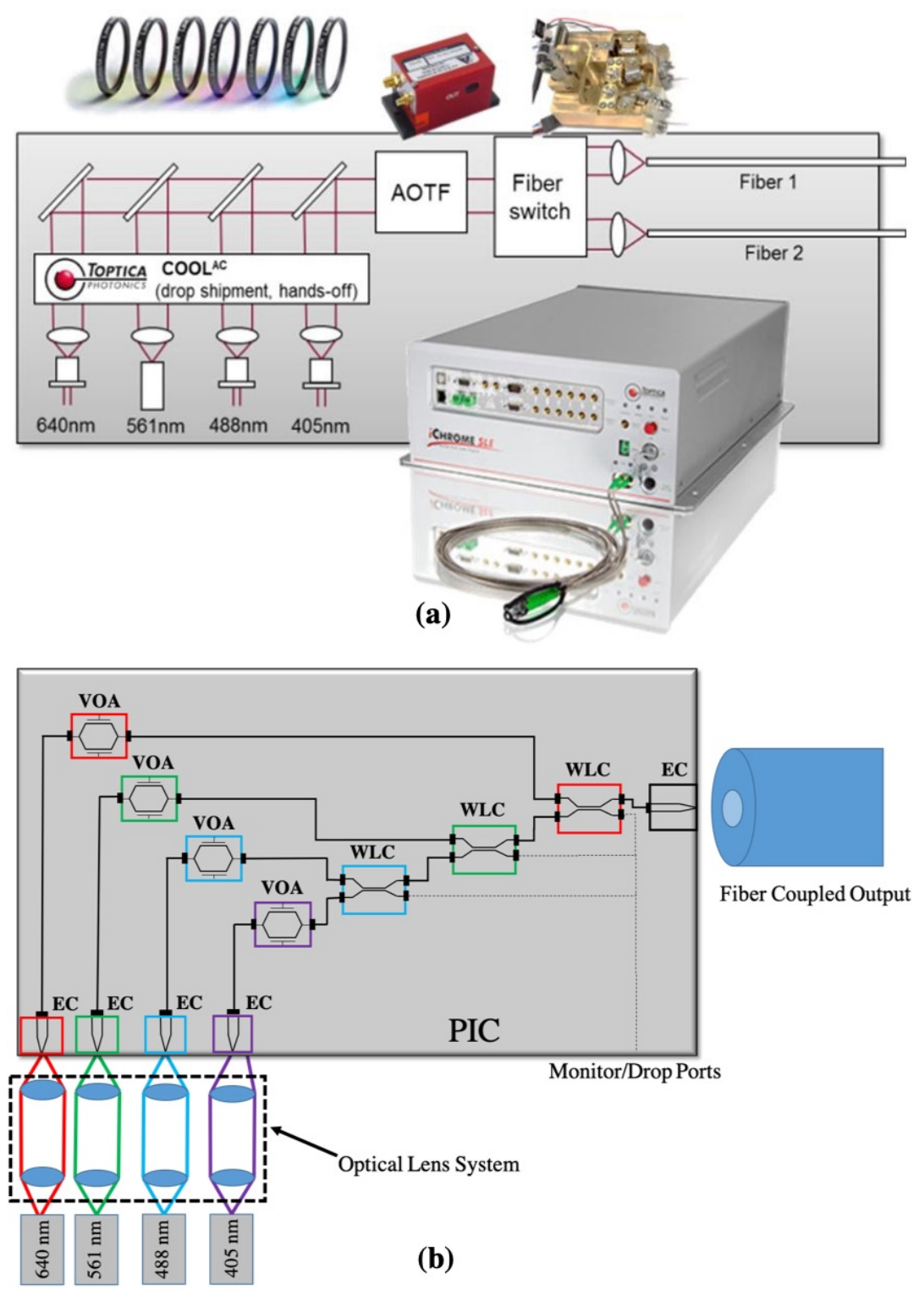

Figure 1. Schematics of (a) a commercially available MLE based on discrete components and (b) a SiN PIC combining the key functionalities. On-chip devices consist in Edge Couplers (EC), Variable Optical Attenuators (VOA) and Wavelength Combiners (WLC).

\subsection{PIC layout \& Mechanical Stage}

Figures 2(a) and 2(b) show the layout of the chip introduced above as well as the mechanical design of the full module. Beams from the four lasers are individually free space coupled to the PIC with help of a lens system. Three wavelengths $(405 \mathrm{~nm}, 488 \mathrm{~nm}$ and $640 \mathrm{~nm}$ ) are directly generated by semiconductor laser diodes; the remaining one, $561 \mathrm{~nm}$, is generated by a $1122 \mathrm{~nm}$ laser diode whose emission is subsequently frequency doubled, referred to as a frequency doubled diode laser (FDDL). An additional mirror serves to steer the beam from the FDDL to the PIC. For $488 \mathrm{~nm}, 561 \mathrm{~nm}$ and $640 \mathrm{~nm}$, a pair of convex lenses proved sufficient to collimate the beam and later focus it onto the corresponding edge coupler (details can be found in section 3.1 describing edge coupler interfaces). 

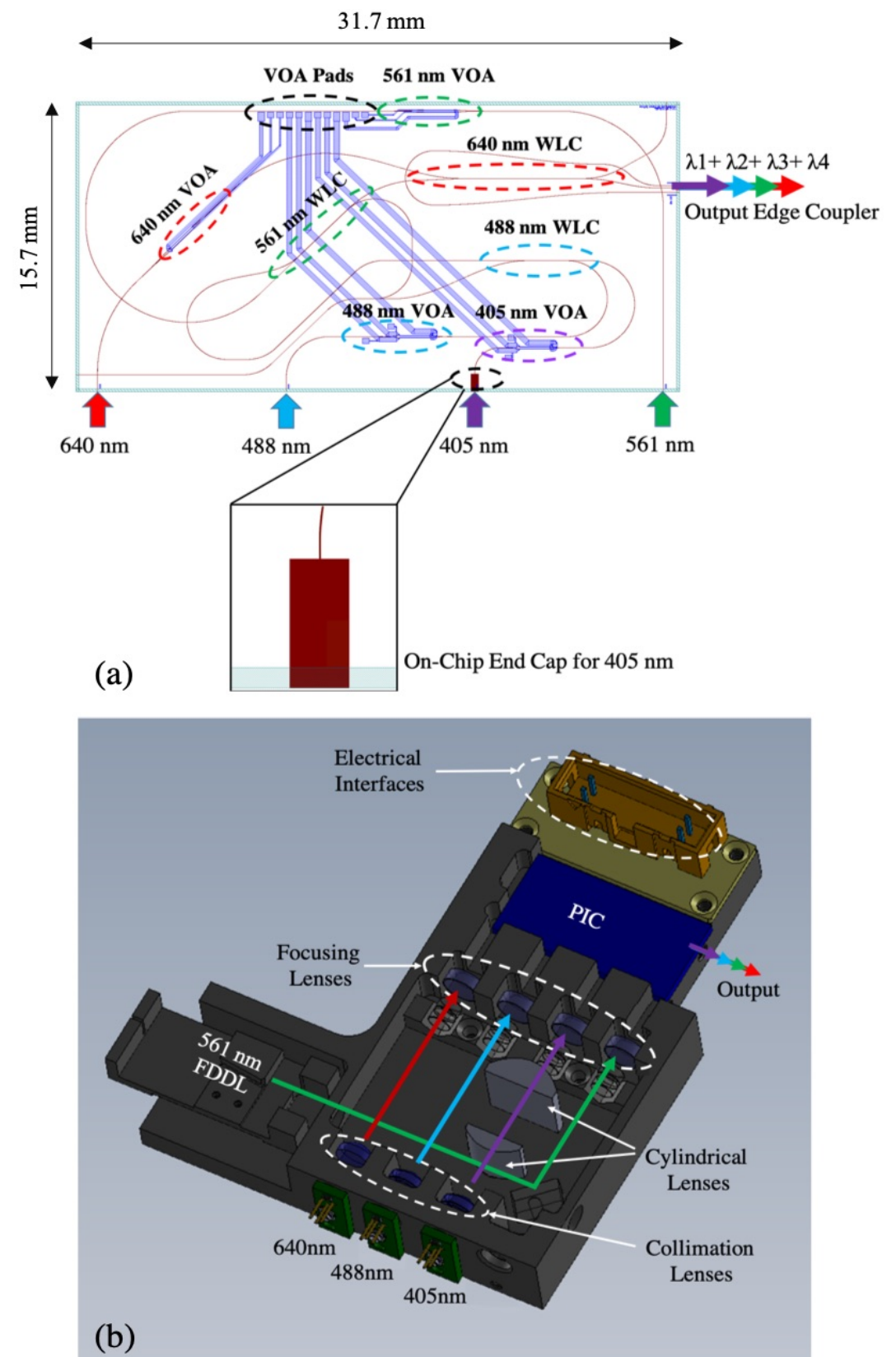

Figure 2. (a) Layout of the PIC and of the on-chip end cap for $405 \mathrm{~nm}$ (inset). (b) Schematic of the mechanical stage for module assembly.

For $405 \mathrm{~nm}$ on the other hand, a different input interface was developed. Chip and fiber interfaces exposed to near-UV short wavelength high intensity radiation suffer from rapid degradation due to photo-assisted contamination, in particular via carbon contamination ${ }^{28}$. To slow down facet degradation, optical fibers can be fused to a short section of coreless fiber. The beam can then be focused to the point inside the fiber at which the coreless section transitions to the single-mode core. Optical intensities at the air interface can be reduced two to three orders of magnitude ${ }^{29}$, greatly improving the reliability of the fiber. Here, a 2D equivalent of this coupling scheme is implemented by transitioning a single-mode waveguide receiving the $405 \mathrm{~nm}$ radiation to a multi-mode waveguide close to the interface (see inset of Fig. 2(a)). An additional pair of cylindrical lenses (see Fig. 2(b)) introduces the right amount of astigmatism to shift the focal point corresponding to focusing in the horizontal plane to inside the PIC, where the multi-mode waveguide transitions into the single-mode waveguide. Attachment of the fiber array at the output of the chip, while not hermetic, provides some amount of sealing, so that the reliability issue was expected to be less severe at this interface. 
The order of the input interfaces was constrained by the requirement to route the longer wavelengths $561 \mathrm{~nm}$ and $640 \mathrm{~nm}$ with bigger waveguide bends to avoid bending losses, the corresponding routing being facilitated by allocating them to the outer input ports (see Fig. 2(a)). The wavelengths $561 \mathrm{~nm}$ and $640 \mathrm{~nm}$ were routed with minimum bending radii of respectively $5.4 \mathrm{~mm}$ and $7 \mathrm{~mm}$. The shorter wavelengths $405 \mathrm{~nm}$ and $488 \mathrm{~nm}$ were routed with minimum bending radii of $1.3 \mathrm{~mm}$ and $1.7 \mathrm{~mm}$. Standard single-mode waveguide widths inside the chip were chosen as $1.2 \mu \mathrm{m}, 1.5 \mu \mathrm{m}, 1.6 \mu \mathrm{m}$, and $1.8 \mu \mathrm{m}$, respectively for $405 \mathrm{~nm}, 488 \mathrm{~nm}, 561 \mathrm{~nm}$, and $640 \mathrm{~nm}$.

\subsection{System chip measurements}

The PIC was characterized using TOPTICA's iChrome MLE sourcing the four required wavelengths. After rotating the polarization to the TM polarization required by our PIC design, light was directly end-fire coupled from a cleaved singlemode fiber to one of the four input interfaces of the chip (while input edge couplers were meant for free space coupling, they have almost the same waveguide width than the output edge coupler, so that resulting insertion losses are identical to the regular packaging scheme with a fiber at the output). The utilized fiber, PANDA type polarization maintaining fiber with a core diameter adjusted to maintain single-mode operation down to $400 \mathrm{~nm}$ (Nufern part number PM-S405-XP) has a $1 / \mathrm{e}^{2}$ Mode Field Diameter (MFD) ranging from $3.3 \mu \mathrm{m}$ at $405 \mathrm{~nm}$ to $4.6 \mu \mathrm{m}$ at $630 \mathrm{~nm}$, sufficiently close to the beam spots formed inside the module for the measured transmission losses to be also representative for the latter (see section on coupling interfaces). At the output of the PIC, light is collected by a microscope objective, transmitted through a polarization beam splitter to monitor and maintain polarization, and finally monitored by a wide area detector. Insertion losses of the receiving apparatus were evaluated by measuring directly the power of a collimated laser beam without interposed PIC and subsequently normalized out from the raw PIC transmission data. As a consequence of the output light collection apparatus not being single mode, insertion losses due to mode mismatch are only occurred once (at the input interface), as opposed to the module in which mode mismatch will play a role at both the input and the output of the PIC. Measurements for $488 \mathrm{~nm}, 561 \mathrm{~nm}$ and $640 \mathrm{~nm}$ were made by injecting light in the corresponding nominal input port and collecting the light from the PIC output. Since the imaging system adapted to couple light into the $405 \mathrm{~nm}$ end cap was not yet available at the time at which these measurements were taken, $405 \mathrm{~nm}$ transmission experiments were done in reverse direction, injecting light into the nominal output port and collecting it from the nominal input. During measurements, the voltage applied to the VOAs was swept from $-15 \mathrm{~V}$ to $15 \mathrm{~V}$, in order to identify the maximum transmission point.

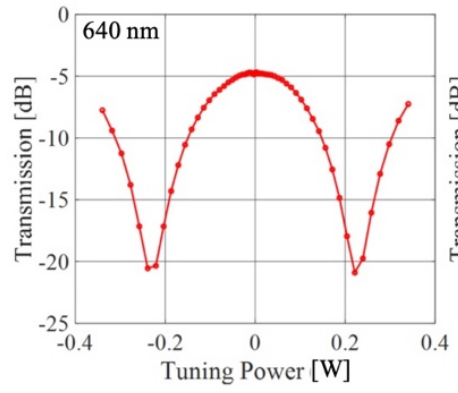

(a)

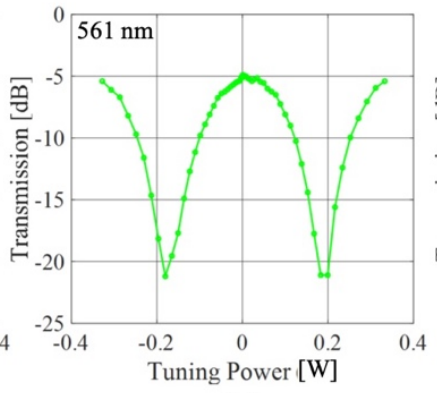

(b)

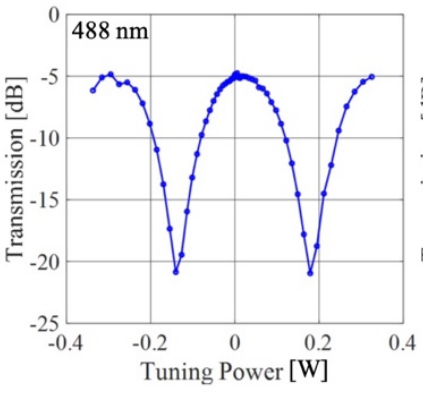

(c)

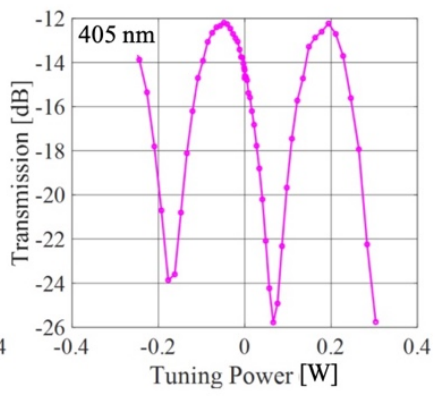

(d)

Figure 3. PIC output (fiber coupled input) during system level characterization of the optical paths for (a) $640 \mathrm{~nm}$, (b) $561 \mathrm{~nm}$, (c) 488 $\mathrm{nm}$. (d) shows the measurements for $405 \mathrm{~nm}$ done in reverse direction, with light injected through the nominal output port. Only one phase shifter is actuated in the MZI-based VOAs at a given time. On the x-axis, a negative power represents a positive power applied to the north phase shifter, while a positive power represents a power applied to the south phase shifter.

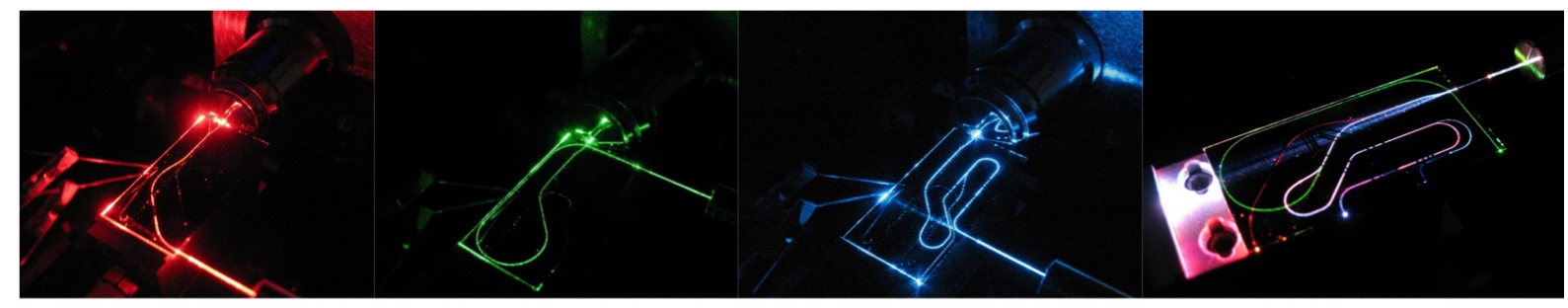

(a)

(b)

(c)

(d)

Figure 4. Photographs of the PIC with (a) $640 \mathrm{~nm}$, (b) $561 \mathrm{~nm}$, and (c) $488 \mathrm{~nm}$ light injected in its respective nominal input port. (d) shows a photograph with all four wavelengths injected through the output. It is clearly visible that the four wavelengths are each routed to their respective nominal input port, showing the (de-)multiplexing functionality. 
Results are shown in Fig. 3. For the three wavelengths $488 \mathrm{~nm}, 561 \mathrm{~nm}$ and $640 \mathrm{~nm}$, the overall PIC transmission losses are close to $5 \mathrm{~dB}$. Measured losses at $405 \mathrm{~nm}$ are larger $(12 \mathrm{~dB})$, in line with the higher edge coupling losses also reported in the following for the chosen edge coupler geometries. These losses correspond to the cumulative effect of the input coupling losses with the insertion losses of the VOA and the cascaded WLCs, and are well in line with device level characterization results reported in Section 3.

Figures 4(a)-4(c) show photographs of the PIC visualizing the waveguides during forward injection of $640 \mathrm{~nm}$ (red), 561 $\mathrm{nm}$ (green) or $488 \mathrm{~nm}$ (blue) light. As previously explained, measurements at $405 \mathrm{~nm}$ (purple) could only be made in reverse direction. Figure 4(d) shows a photograph of the chip with all four wavelengths injected through the nominal output port, illustrating the (de-)multiplexing functionality.

\section{DEVICE LEVEL CHARACTERIZATION}

\subsection{Edge coupler simulation and characterization}

Input edge couplers for the three wavelengths $488 \mathrm{~nm}, 561 \mathrm{~nm}$ and $640 \mathrm{~nm}$ were optimized to maximize free-space incoupling efficiency from the lasers as assisted by a pair of convex lenses, i.e., a collimating lens immediately after the laser and a focusing lens prior to the PIC. Waveguide widths at the input edge couplers were co-optimized with the focal length of the focusing lenses, resulting in the waveguide widths at the edge of the chip to be chosen as $1 \mu \mathrm{m}$ (the minimum feature size in the chosen process) for wavelengths $488 \mathrm{~nm}$ and $561 \mathrm{~nm}$, and as $1.1 \mu \mathrm{m}$ for $640 \mathrm{~nm}$. 1/ $\mathrm{e}^{2}$ half diffraction angles in the in-plane $\left(\theta_{x}\right)$ and out-of-plane $\left(\theta_{z}\right)$ directions were evaluated based on a far-field expansion of mode profiles calculated in the modeling software FIMMWAVE. In order to verify modeling results after chip fabrication, light was injected through the nominal output port as shown in Fig. 4(d) (but one wavelength at a time) and the resulting beams at the nominal input ports measured in the far field. Results are summarized in Table 1. Since the chosen $1 \mu \mathrm{m}$ edge coupler width is well below the single-mode cutoff even at $488 \mathrm{~nm}$ (a consequence of the very thin SiN layer and the resulting low confinement), mode profiles were close to having a circular symmetry and diffraction angles in $\mathrm{x}$ and $\mathrm{z}$ are very close. Moreover, corresponding far field beams had close to Gaussian shapes. Variations of the diffraction angles for different wavelengths were accommodated by choosing focusing lenses with different focal distances. Since the collimated beams being focused had different dimensions in the $\mathrm{x}$ - and z-directions as a result of the laser diode emission profiles, a tradeoff had to be struck in the chosen focal distances, resulting in modest additional excess insertion losses estimated to be 1.3 $\mathrm{dB}$ to $1.4 \mathrm{~dB}$ when using the selected, commercially available lenses.

Table 1. Simulated and measured far field $1 / \mathrm{e}^{2}$ half-diffraction-angles.

\begin{tabular}{|l|l|l|l|l|}
\hline Wavelength & Sim. $\boldsymbol{\theta}_{\boldsymbol{x}}$ & Sim. $\boldsymbol{\theta}_{\boldsymbol{z}}$ & Meas. $\boldsymbol{\theta}_{\boldsymbol{x}}$ & Meas. $\boldsymbol{\theta}_{\boldsymbol{z}}$ \\
\hline $405 \mathrm{~nm}$ (diode, end cap) & $9.55^{\circ}$ & $15.93^{\circ}$ & $9.29^{\circ}$ & $7.54^{\circ}$ \\
\hline $488 \mathrm{~nm}$ (diode) & $6.85^{\circ}$ & $7.14^{\circ}$ & $6.84^{\circ}$ & $7.29^{\circ}$ \\
\hline $561 \mathrm{~nm}$ (FDDL) & $5.17^{\circ}$ & $5.36^{\circ}$ & $5.9^{\circ}$ & $6.3^{\circ}$ \\
\hline $640 \mathrm{~nm}$ (diode) & $4.42^{\circ}$ & $4.44^{\circ}$ & $3.66^{\circ}$ & $3.89^{\circ}$ \\
\hline
\end{tabular}

As previously mentioned, the $405 \mathrm{~nm}$ input interface looks very different due to the need of providing an end cap. A schematic of the interface is shown in Fig. 5. A $1 \mu \mathrm{m}$ wide waveguide transitions into an unetched slab region wide enough for the beam to freely diffract in the plane of the chip (when propagating in reverse direction). The slab region is $803 \mu \mathrm{m}$ long before reaching the edge of the chip, at which the beam is simulated to have diffracted to reach an MFD of $180 \mu \mathrm{m}$, corresponding to a $1 / \mathrm{e}^{2}$ diffraction half-angle of $6.46^{\circ}$ inside the slab (with effective index $n_{\text {eff }}=1.48$ at $405 \mathrm{~nm}$ ). At the output of the PIC, Snell's law results in the beam diffraction half angle increasing to $9.55^{\circ}$. Expansion inside the slab corresponds to a factor 116 relative to the mode's $1.55 \mu \mathrm{m}$ MFD inside the $1 \mu \mathrm{m}$ waveguide. The actual in-plane diffraction half-angle outside of the chip was measured as $9.29^{\circ}$, in close agreement with the calculated value. Due to the change of direction of the rays as they exit the chip, the virtual focus point of the beam, for the in-plane beam shape, is $\sim 540 \mu \mathrm{m}$ inside the chip, creating a large astigmatism since the beam remains confined in the z-direction until it reaches the facet. In the z-direction, the beam's $1 / \mathrm{e}^{2}$ half-diffraction angle is simulated to be $15.93^{\circ}$ and measured to be $7.54^{\circ}$. The substantial difference between measured and simulated values might point to a discrepancy in assumed layer thicknesses or refractive index. It is also expected to be at least partially due to the abrupt interface between the $1 \mu \mathrm{m}$ wide waveguide and the slab: Since $1 \mu \mathrm{m}$ is below the single-mode cutoff region for the chosen layer thicknesses, and thus relatively small even for 405 
$\mathrm{nm}$, the mode in the waveguide is more delocalized in the vertical direction than in the slab (as is also the case in inverse tapers). At the abrupt interface, some of the light may be converted into cladding modes, modifying the emission profile at the chip facet. Consequently, it would have been beneficial to first taper the waveguide to a wider width, even though this would have resulted in a reduction of the in-plane diffraction angle and thus reduce the effectiveness of the end cap.

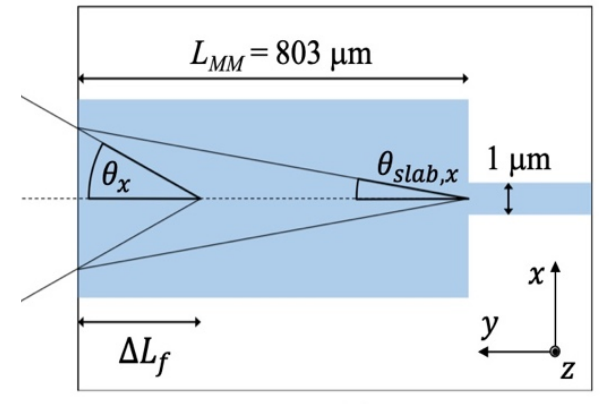

(a)

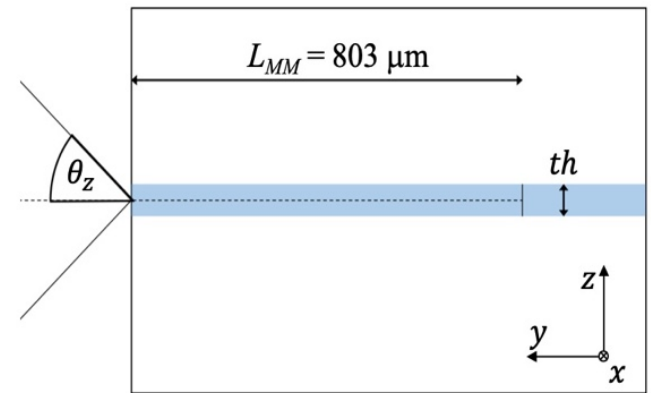

(b)

Figure 5. $405 \mathrm{~nm}$ edge coupler with 2D end cap. (a) shows the top view and (b) the side view.

Due to the confinement in the vertical direction, the effective beam height at the edge of the chip, defined as the ratio of the light intensity integrated along the z-direction at the center of the slab $(x=0)$ divided by the maximum intensity, is only $0.45 \mu \mathrm{m}$. Together with the effective beam width $w_{\text {eff }}=\sqrt{\pi / 2} \cdot(M F D / 2)$, determined by the MFD of $180 \mu \mathrm{m}$, this results in an effective beam size of $\sim 50 \mu \mathrm{m}^{2}$. This is only a factor $\sim 10$ larger than the typical mode size of single-mode fibers at $405 \mathrm{~nm}\left(\sim 4.3 \mu \mathrm{m}^{2}\right)$. Compared to the 2-3 orders of magnitude intensity reduction typically obtained with coreless fiber end caps $^{29}$, this is only a modest improvement, so that reliability concerns remain. We have made a long-term reliability test on a similar chip with an identical $405 \mathrm{~nm}$ input interface and a fiber array attached to the $1 \mu \mathrm{m}$ wide output waveguide ( $\sim 0.56 \mu \mathrm{m}^{2}$ effective mode area), exposing the $405 \mathrm{~nm}$ input facet to a continuous wave $20 \mathrm{~mW}$ beam shaped for optimum in-coupling efficiency. Results can be seen in Fig. 6. After 75 hours of continuous exposure the power collected in the output face dropped to half its initial value, pointing to reliability at $405 \mathrm{~nm}$ requiring further work. A possible path forward might consist in 2D beam expansion in coreless fibers at the input as well as sealing of the output fiber interface.
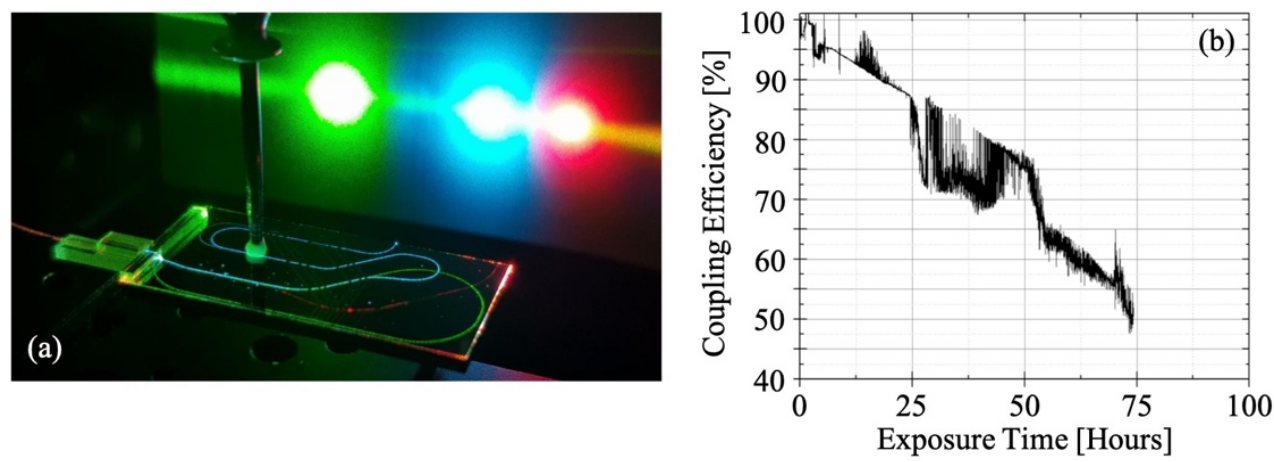

Figure 6. (a) Chip with permanently attached fiber array used for long term reliability test. (b) Transmission of $405 \mathrm{~nm}$ through the chip and fiber array during a long time exposure test with $405 \mathrm{~nm}$ radiation (coupled in through the nominal $405 \mathrm{~nm}$ input interface with on-chip end cap). Transmission drops to $50 \%$ of its initial value after 75 hours exposure.

As already mentioned, the output interface of the chip is meant to be coupled to an optical fiber with reduced core dimensions to remain single mode in the visible. Test structures were used to measure the interface losses between the aforementioned PM-S405-XP optical fiber and edge couplers with varying waveguide widths. Reported losses only correspond to mode mismatch at the fiber to chip interface of the test structure (in which light is injected in this experiment), since light is collected on the other side with free space optics and a large area detector. Results are shown in Fig. 7. It is apparent that the smallest allowed waveguide width, $1 \mu \mathrm{m}$, is best for the longer wavelengths $488 \mathrm{~nm}, 561 \mathrm{~nm}$ and 640 $\mathrm{nm}$, for which the edge coupler acts as an inverse taper (blowing up the field due to reduced confinement as the waveguide width is narrowed). It is also apparent that this coupling mechanism does not work at $405 \mathrm{~nm}$ given the minimum feature size. Rather, at $405 \mathrm{~nm}$ the optimum is obtained for a 3-4 $\mu \mathrm{m}$ wide waveguide, acting as a regular taper increasing the waveguide mode in one dimension as the waveguide is widened. Since all the wavelengths are routed out through a single 
output edge coupler, a trade-off has to be struck. While the 3-4 $\mu \mathrm{m}$ waveguide width would have resulted in the best overall performance as monitored over all wavelengths, we opted for a $1 \mu \mathrm{m}$ wide taper in this chip, giving the best performance for 3 out of the 4 wavelengths. $488 \mathrm{~nm}, 561 \mathrm{~nm}$ and $640 \mathrm{~nm}$ all have insertion losses in the 1-2 dB range, while $405 \mathrm{~nm}$ suffers from substantially higher $8 \mathrm{~dB}$ insertion losses. These additional 6 to $7 \mathrm{~dB}$ are also seen in the overall system-chip level characteristics, since overall insertion losses for the three longer wavelengths were $5 \mathrm{~dB}$, while the $405 \mathrm{~nm}$ optical path suffered from a total of $12 \mathrm{~dB}, 4$ of which could be straightforwardly recovered by resizing the edge coupler.

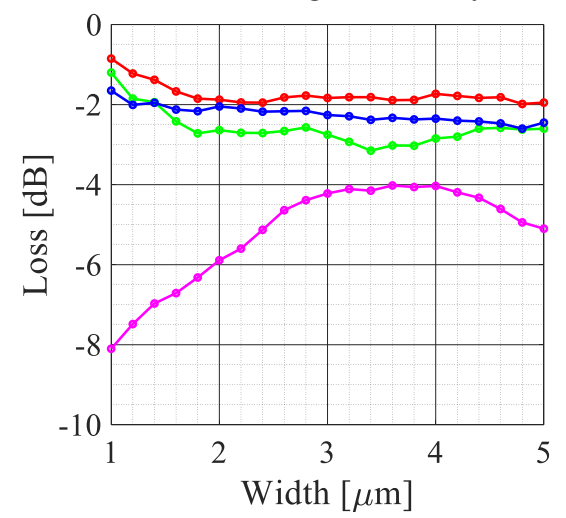

Figure 7. Characterization results of modeled edge couplers for optimization of coupling loss for all wavelengths. Curves are color coded according to the wavelength (red: $640 \mathrm{~nm}$, green: $561 \mathrm{~nm}$, blue: $488 \mathrm{~nm}$, purple: $405 \mathrm{~nm}$ ).

\subsection{Wavelength Combiner (WLC)}

Due to the wide wavelength spacing between the four channels, wavelength division multiplexers as typically used in communications such as arrayed waveguide gratings or echelle gratings are not suitable here. Moreover, MZI based interleavers also pose challenges due to the irregular wavelength spacing. The wavelength combiners used here are implemented in the form of Directional Couplers (DC) that leverage the wavelength dependence of the modal confinement. The basic concept of a single WLC stage is illustrated in Fig. 8(b). The waveguide width used inside the DC is chosen such that the shorter wavelength(s), in this case $405 \mathrm{~nm}$, is highly confined and does not couple to the other waveguide. The waveguide width is however also chosen such that the longer wavelength, here $488 \mathrm{~nm}$, has a sufficiently wide evanescent field to couple over (to achieve these two properties jointly, the large wavelength spacing now turns into an advantage). The shorter wavelength, $405 \mathrm{~nm}$, enters the DC through the "main" waveguide that continues to further processing stages at the output of the DC. The longer wavelengths enter the DC through the other "add" waveguide and are coupled over to the main bus waveguide. This WLC architecture is relatively robust: Inside the coupling region, both waveguides have identical cross-sections, i.e., phase matching inside the DC is guaranteed. The main constraint is on the coupler length to achieve full coupling of the added, longer wavelength (with Fig. 9 giving some sense on tolerances). To obtain repeatable results, precise control of the SiN layer thickness is of paramount importance.

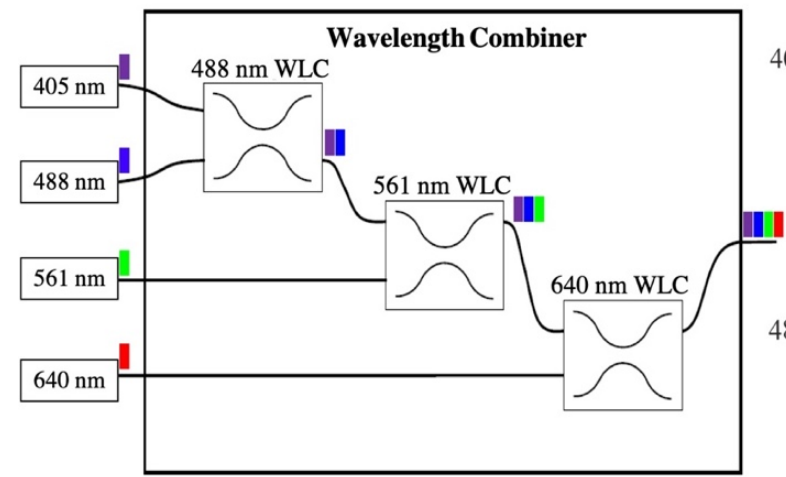

(a)

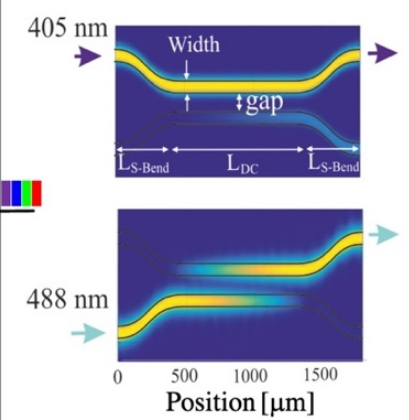

(b)

Figure 8. (a) Schematics of the three cascaded WLC stages. (b) Simulation results for the first stage adding $488 \mathrm{~nm}$ light to the bus already carrying $405 \mathrm{~nm}$ light. 
Multiplexer stages are named after the wavelength that is being added. After adding $488 \mathrm{~nm}$ to $405 \mathrm{~nm}$ (488 nm WLC), $561 \mathrm{~nm}$ is added to the latter two (561 nm WLC), and finally $640 \mathrm{~nm}$ is added to the first three (640 nm WLC). Importantly, possible insertion losses due to layer thickness variations and/or bad sizing of the device length are incurred only once by each of the added wavelengths $488 \mathrm{~nm}, 561 \mathrm{~nm}$ and $640 \mathrm{~nm}$, since transmission of the shorter wavelengths through the "main" waveguide is very robust in this configuration (essentially, they do not interact with the other waveguide).

Each wavelength combiner is made out of two S-bends and a straight directional coupler section, with waveguide width, spacing and length optimized for each stage. Table 2 summarizes the characteristics of the three WLC stages implemented in the system chip.

Table 2. Nominal selected geometrical features of WLC stages for adding $488 \mathrm{~nm}, 561 \mathrm{~nm}$, and $640 \mathrm{~nm}$.

WG gap refers to the WG spacing, measured edge to edge.

\begin{tabular}{|l|l|l|l|}
\hline WLC Stage $\rightarrow$ & \multicolumn{1}{|c|}{ Add 488 nm } & Add 561 nm & Add 640 nm \\
\hline WG Width & $1.5 \mu \mathrm{m}$ & $1 \mu \mathrm{m}$ & $1.5 \mu \mathrm{m}$ \\
\hline WG Gap & $1.8 \mu \mathrm{m}$ & $4.6 \mu \mathrm{m}$ & $6 \mu \mathrm{m}$ \\
\hline $\begin{array}{l}\text { Linear DC } \\
\text { Section }\end{array}$ & $1239 \mu \mathrm{m}$ & $2324 \mu \mathrm{m}$ & $5114 \mu \mathrm{m}$ \\
\hline $\begin{array}{l}\text { Length incl. } \\
\text { S-bends }\end{array}$ & $2139 \mu \mathrm{m}$ & $3224 \mu \mathrm{m}$ & $6014 \mu \mathrm{m}$ \\
\hline
\end{tabular}

Figure 9 shows results for the " $488 \mathrm{~nm}$ WLC" adding $488 \mathrm{~nm}$ to $405 \mathrm{~nm}$ and for the " $561 \mathrm{~nm}$ WLC" adding $561 \mathrm{~nm}$ to the combined $405 \mathrm{~nm}$ and $488 \mathrm{~nm}$ as a function of the linear DC section length, that was varied in separated test structures used for component characterization and development (S-bends were kept constant and measure $450 \mu \mathrm{m}$ on both sides of the DC). It is apparent that the shorter wavelength, $405 \mathrm{~nm}$ for " $488 \mathrm{~nm}$ WLC" and $488 \mathrm{~nm}$ for "561 nm WLC", remains in the main waveguide to near $100 \%$. Some amount is measured in the complementary DC output, but since this amount appears to be independent of the length of the DC, it is attributed primarily to stray light in the test structure. The longer wavelength, $488 \mathrm{~nm}$ in "488 nm WLC" and $561 \mathrm{~nm}$ in "561 nm WLC", is coupled over from the complementary input to the main waveguide, with a coupling strength depending on the length of the junction, as expected in a DC. The finite extinction seen in the characterization data is also partially attributed to a test artefact due to stray light. Phase matching should be obtained by design as the junctions are designed to be fully symmetric. It is apparent that the lengths chosen in the system chip were slightly below the optimum determined from the test structures, due to a small overestimation of the coupling strengths, so that 1 to $1.5 \mathrm{~dB}$ of the total insertion losses for the $488 \mathrm{~nm}$ and $561 \mathrm{~nm}$ paths are attributed to this.

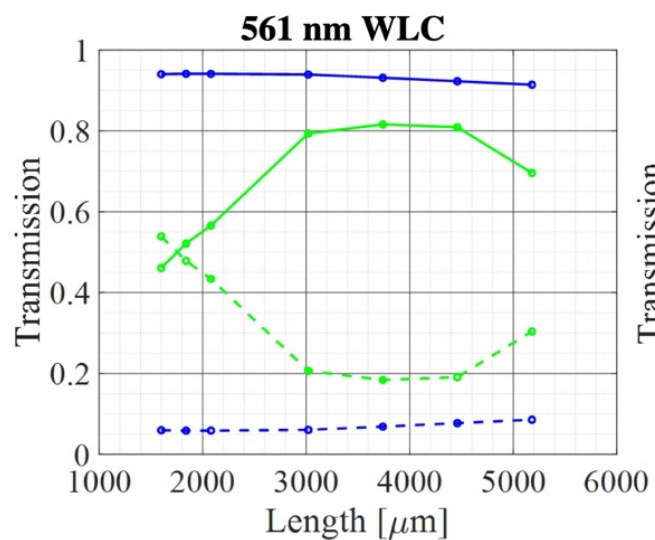

(a)

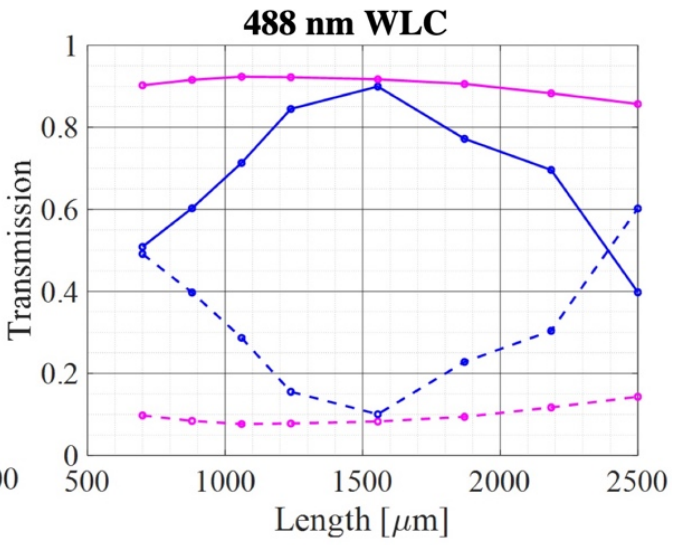

(b)

Figure 9. Characterization of (a) the $561 \mathrm{~nm}$ WLC and (b) the $488 \mathrm{~nm}$ WLC for varying junction lengths. The $\mathrm{x}$-axes show the length of the linear section of the junctions (the length of the S-bends were kept constant and have to be added). Solid and dashed lines show the power exiting the WLC through the main bus waveguide and the add waveguide. Curves are color coded according to wavelength (green: $561 \mathrm{~nm}$, blue: $488 \mathrm{~nm}$, purple: $405 \mathrm{~nm}$ ). 


\subsection{Variable Optical Attenuator}

VOAs were implemented in the form of MZIs with thermally tuned phase shifters (Fig. 10). 1x2 splitters at the input and output of the MZIs were implemented with Multi-Mode Interferometers (MMIs) that had to be individually designed for each of the four wavelengths. Geometric characteristics of the MMI layouts are summarized in Table 3.

Heaters in the phase shifters were designed as $20 \mu \mathrm{m}$ wide and $1500 \mu \mathrm{m}$ long metal resistors. The resistance of the resistors was consistently measured to be $726 \pm 26 \Omega$ (with the variation at least partially attributed to resistive losses in the metal lines connecting the pad frame with the heaters, as the variations correlate with their length). The electrical power required to achieve a $\pi$ phase shift $\left(P_{\pi}\right)$ is extracted from the data shown in Fig. 3. It features a clear dependence on wavelength (exceeding by far the scatter of the resistance data), which we attribute to the wavelength dependence of the thermo-optic coefficients of the materials. The extracted values of $P_{\pi}$ are $238 \mathrm{~mW}, 314 \mathrm{~mW}, 368 \mathrm{~mW}$, and $456 \mathrm{~mW}$, respectively at 405 $\mathrm{nm}, 488 \mathrm{~nm}, 561 \mathrm{~nm}$ and $640 \mathrm{~nm}$.

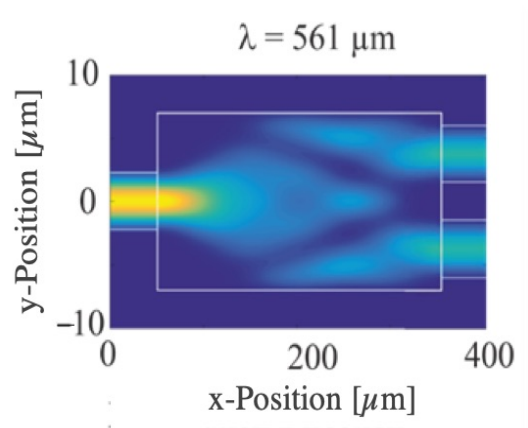

(a)

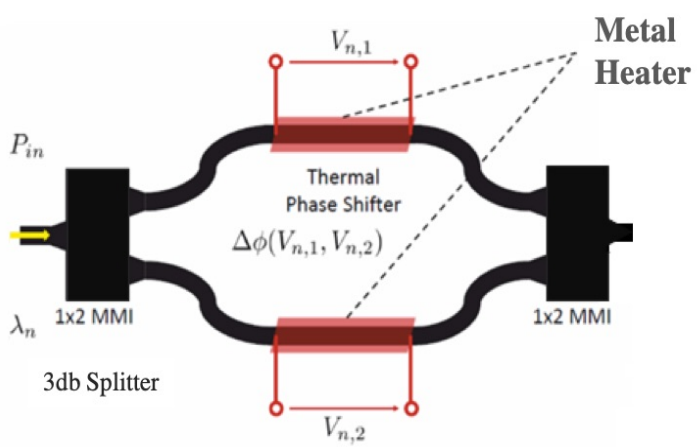

(b)

Figure 10. (a) $3 \mathrm{~dB}$ splitters are implemented as 1x2 MMIs independently optimized for each wavelength (shown here for 561 $\mathrm{nm}$ ). (b) Schematics of a VOA structure realized as a thermally tuned MZI, with metal resistors fabricated on top of the upper cladding overlaying the waveguides.

Table 3. Layout parameters of the 1 by 2 MMIs used in the VOAs. "Offset of output waveguides" refers to the displacement of the center of the output waveguides relative to the center of the MMI section.

\begin{tabular}{|l|l|l|l|l|}
\hline Wavelength & \multicolumn{1}{|c|}{$\begin{array}{c}\text { Width of } \\
\text { MMI Section }\end{array}$} & $\begin{array}{c}\text { Length of } \\
\text { MMI Section }\end{array}$ & $\begin{array}{c}\text { Width of Input } \\
\text { \& Output } \\
\text { Waveguides }\end{array}$ & $\begin{array}{c}\text { Offset of } \\
\text { Output } \\
\text { Waveguides }\end{array}$ \\
\hline $405 \mathrm{~nm}$ & $13.2 \mu \mathrm{m}$ & $349.5 \mu \mathrm{m}$ & $3.6 \mu \mathrm{m}$ & $3.45 \mu \mathrm{m}$ \\
\hline $488 \mathrm{~nm}$ & $12 \mu \mathrm{m}$ & $253.1 \mu \mathrm{m}$ & $3.5 \mu \mathrm{m}$ & $3.25 \mu \mathrm{m}$ \\
\hline $561 \mathrm{~nm}$ & $14 \mu \mathrm{m}$ & $306.1 \mu \mathrm{m}$ & $4.5 \mu \mathrm{m}$ & $3.75 \mu \mathrm{m}$ \\
\hline $640 \mathrm{~nm}$ & $19 \mu \mathrm{m}$ & $490.0 \mu \mathrm{m}$ & $6.1 \mu \mathrm{m}$ & $5.15 \mu \mathrm{m}$ \\
\hline
\end{tabular}

\section{CONCLUSION \& PROSPECTS}

We have presented device and chip level characterization results for a PIC incorporating core functionalities for the realization of a miniaturized multi-color laser engine. The chip was implemented in the TriPlex ${ }^{\mathrm{TM}}$ silicon nitride technology in the framework of the PIX4life pilot line, with layer thicknesses optimized for visible wavelength life-science applications. The demonstrated chip combines variable optical attenuation and multiplexing of four wavelengths covering the entire visible spectrum $(405 \mathrm{~nm}, 488 \mathrm{~nm}, 561 \mathrm{~nm}, 640 \mathrm{~nm})$ with moderate overall insertion losses $(5 \mathrm{~dB})$ for three of the wavelengths $(488 \mathrm{~nm}, 561 \mathrm{~nm}, 640 \mathrm{~nm})$. Some work remains to be done to improve both insertion losses and reliability of the $405 \mathrm{~nm}$ interfaces. A chip with extended functionality combining variable optical attenuation with switching between two output fibers (to switch e.g. between confocal and flood illumination) has been fabricated and is being characterized. A version of the chip including beam shaping in its output stage for direct free space coupling into a flow cytometer is also in the process of being mounted in a demonstrator module. Results pertaining to these additional developments further leveraging the capabilities of the PIC technology will be reported in the future. 


\section{ACKNOWLEDGEMENTS}

This work was supported by the European Union's Horizon 2020 research and innovation program under grant agreement no. 688519 (PIX4life).

\section{REFERENCES}

[1] Blumenthal, D. J., Heideman, R., Geuzebroek, D., Leinse, A., and Roeloffzen, C., "Silicon Nitride in Silicon Photonics," Proceedings of the IEEE 106(12), 2209-2231 (2018).

[2] Muñoz, P., Micó, G., Bru, L. A., Pastor, D., Pérez, D., Doménech, J. D., Fernández, J., Baños, R., Gargallo, B., Alemany, R., Sánchez, A. M., Cirera, J. M., Mas, R., and Domínguez, C., "Silicon Nitride Photonic Integration Platforms for Visible, Near-Infrared and Mid-Infrared Applications," Sensors 17(9), 2088 (2017).

[3] Dhakal, A., Wuytens, P. C., Peyskens, F., Jans, K., Le Thomas, N., and Baets, R., "Nanophotonic Waveguide Enhanced Raman Spectroscopy of Biological Submonolayers," ACS Photon. 3, 2141-2149 (2016).

[4] Romero-García, S., Merget, F., Zhong, F., Finkelstein, H., Witzens, J., "Silicon nitride CMOS-compatible platform for integrated photonics applications at visible wavelengths," Opt. Expr. 21(12), 14036-14046 (2013).

[5] Duval, D., Lechuga, L. M., "Breakthroughs in Photonics 2012: 2012 Breakthroughs in Lab-on-a-Chip and Optical Biosensors," Lab Chip 12, 1987-1994 (2012).

[6] Soref, R., "Mid-infrared photonics in silicon and Germanium," Nat. Phot. 4, 495-497 (2010).

[7] Dhakal, A., Wuytens, P., Raza, A., Le Thomas, N., Baets, R., "Silicon Nitride Background in Nanophotonic Waveguide Enhanced Raman Spectroscopy," Mat. 10(2), 140 (2017).

[8] Spencer, D. T., Bauters, J. F., Heck, M. J. R., and Bowers, J. E., "Integrated waveguide coupled $\mathrm{Si}_{3} \mathrm{~N}_{4}$ resonators in the ultrahigh-Q regime," Optica 1(3), 153-157 (2014).

[9] Pfeifer, M. H. P., Kordts, A., Brasch, V., Zervas, M., Geiselmann, M., Jost, J. D., and Kippenberg, T. J., "Photonic Damascene process for integrated high-Q microresonator based nonlinear photonics," Optica 3(1), 20-25 (2016).

[10]Levy, J. S., Gondarenko, A., Foster, M. A., Turner-Foster, A. C., Gaeta, A. L., and Lipson, M., "CMOScompatible multiple-wavelength oscillator for on-chip optical interconnects," Nat. Photon. 4(1), 37-40 (2010).

[11] Wang, L., Chang, L., Volet, N., Pfeiffer, M. H. P., Zervas, M., Guo, H., Kippenberg, T. J., and Bowers, J. E., "Frequency comb generation in the green using silicon nitride microresonators," Las. Photon. Rev. 10(4), 631638 (2016).

[12] Daldosso, N., Melchiorri, M., Riboli, F., Girardini, M., Pucker, G., Crivellari, M., Bellutti, P., Lui, A., and Pavesi, L., "Comparison Among Various Si3N4 Waveguide Geometries Grown Within a CMOS Fabrication Pilot Line," J. Lightw. Technol. 22, 1734-1740 (2004).

[13]Iqbal, M., Gleeson, M. A., Spaugh, B., Tybor, F., Gunn, W. G., Hochberg, M., Baehr-Jones, T., Bailey, R. C., and Gunn, L. C., "Label-Free Biosensor Arrays Based on Silicon Ring Resonators and High-Speed Optical Scanning Instrumentation,” J. Sel. Top. Quant. Electron. 16(3), 654-661 (2010).

[14] Nägerl, U. V., Willig, K. I., Hein, B., Hell, S. W., and Bonhoeffer, T., "Live-cell imaging of dendritic spines by STED microscopy," Proc. Nat. Acc. Sci 105(48), 18982-18987 (2008).

[15] Perfetto, S. P., Chattopadhyay, P. K., and Roederer, M., "Seventeen-colour flow cytometry: unravelling the immunesystem," Nat. Rev. Immu. 4(8), 648-655 (2004).

[16] Kampasi, K., Stark, E., Seymour, J., Na, K., Winful, H. G., Buzsáki, G., Wise, K. D., and Yoon, E., “Fiberless multicolor neural optoelectrode for in vivo circuit analysis," Sci. Rep. 6, 30961 (2016).

[17] Romero-García, S., Klos, T., Klein, E., Leuermann, J., Geuzebroek, D., van Kerkhof, J., Büscher, M., Krieg, J., Leisching, P., Witzens, J., "Photonic integrated circuits for multi-color laser engines," Proc. SPIE 10108, $101080 Z$ (2017).

[18] Wörhoff, K., Heidemann, R. G., Leinse, A., and Hoekman, M., "TriPleX: a versatile dielectric photonic platform," Adv. Opt. Techn. 4(2), 189-207 (2015).

[19] Lax, M., "Classical Noise V. Noise in Self-Sustained Oscillators," Phys. Rev. 160(2) 290-307 (1967).

[20] Gorin, A., Jaouad, A., Grondin, E., Aimez, V., and Charette, P., "Fabrication of silicon nitride waveguides for Visible-light using PECVD: a study of the effect of plasma frequency on optical properties," Opt. Expr. 16(18), 13509-13516 (2008).

[21] www.pix4life.eu 
[22] Pérez, D., Fernández, J., Baños, R., Doménech, J. D., Sánchez, A. M., Cirera, J. M., Mas, R., Sánchez, J., Durán, S., Pardo, E., Domínguez, C., Pastor, D., Capmany, J., and Muñoz, P., "Thermal tuners on a Silicon Nitride platform," arXiv:1604.02958 (2016).

[23] Hosseini, N., Dekker, R., Hoekman, M., Dekkers, M., Bos, J., Leinse, A., and Heideman, R., "Stress-optic modulator inTriPleX platform using a piezoelectric lead zirconate titanate (PZT) thin film," Opt. Expr. 23(11), 14018-14026 (2015).

[24] Alexander, K., George, J. P., Verbist, J., Neyts, K., Kuyken, B., Van Thourhout, D., and Beeckman, J., "Nanophotonic Pockels modulators on a silicon nitride platform," Nat. Comm. 9, 3444 (2018).

[25] Romero-García, S., Leuermann, J., Merget, F., Klos, T., Koch, S., Leishing, P., Klein, E., Geuzebroek, D., Dekker, R., van Kerkhof, J., Krieg, J., Büscher, M., Witzens, J., "Silicon Nitride Photonic Integrated Circuits for Multi-Color Optical Engines with Application in Flow Cytometry," Proc. IEEE Photon. Soc. Sum. Top. Meet., 219-221 (2017).

[26] www.toptica.com/technology/toptica-proprietary/cool/

[27] Geuzebroek, D., Dekker, R., van Dijk, P., "Photonics Packaging Made Visible," Optik\&Photonik 12(5), 34-38 (2017).

[28] Kim, C. C., Choi, Y., Jang, Y. H., Kang, M. K., Joo, M., and Noh, M. S., "Degradation modes of high-power InGaN/GaN laser diodes on low-defect GaN substrates," Proc. SPIE 6894, 68940 O (2008).

[29] Knigge, A., Knothe, C., Oechsner, U., and Federau, G., "Fibers with End Caps,” Physics' Best, 2-5 (2017). 